\title{
Electronic Supplementary Material
}

\section{$\mathrm{Bi}_{2} \mathrm{WO}_{6}$ quantum dot-intercalated ultrathin montmo- rillonite nanostructure and its enhanced photocatalytic performance}

Songmei Sun ${ }^{1}$, Wenzhong Wang ${ }^{1}(\bowtie)$, Dong Jiang ${ }^{1}$, Ling Zhang ${ }^{1}$, Xiaoman $\mathrm{Li}^{1}$, Yali Zheng ${ }^{1}$, and Qi $\mathrm{An}^{2}$

'State Key Laboratory of High Performance Ceramics and Superfine Microstructure, Shanghai Institute of Ceramics, Chinese Academy of Sciences, Shanghai 200050, China

${ }^{2}$ Materials and Process Simulation Center, California Institute of Technology, Pasadena, CA 91125, USA

Supporting information to DOI 10.1007/s12274-014-0511-2
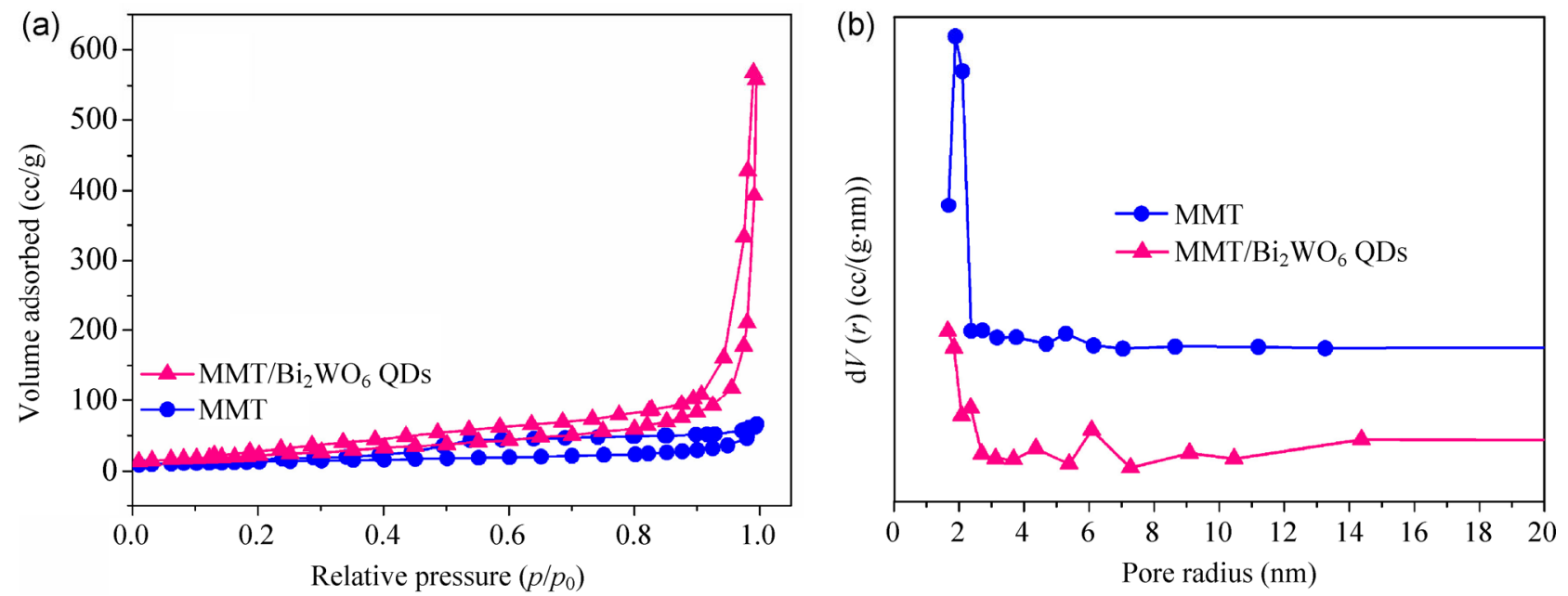

Figure S1 (a) Typical $\mathrm{N}_{2}$ adsorption-desorption isotherms of pure MMT and MMT/Bi $2 \mathrm{WO}_{6}$ QDs; (b) the corresponding pore-size distribution.

Address correspondence to wzwang@mail.sic.ac.cn 